\title{
Microbial strain improvement for enhanced polygalacturonase production by Aspergillus sojae
}

\author{
Doreen Heerd • Canan Tari • Marcelo Fernández-Lahore
}

Received: 9 December 2013 / Revised: 28 January 2014 / Accepted: 2 March 2014 / Published online: 4 April 2014

(C) Springer-Verlag Berlin Heidelberg 2014

\begin{abstract}
Strain improvement is a powerful tool in commercial development of microbial fermentation processes. Strains of Aspergillus sojae which were previously identified as polygalacturonase producers were subjected to the costeffective mutagenesis and selection method, the so-called random screening. Physical (ultraviolet irradiation at $254 \mathrm{~nm})$ and chemical mutagens $(N$-methyl- $N$ '-nitro- $N$ nitrosoguanidine) were used in the development and implementation of a classical mutation and selection strategy for the improved production of pectic acid-degrading enzymes. Three mutation cycles of both mutagenic treatments and also the combination of them were performed to generate mutants descending from $A$. sojae ATCC 20235 and mutants of A. sojae CBS 100928. Pectinolytic enzyme production of the mutants was compared to their wild types in submerged and solid-state fermentation. Comparing both strains, higher pectinase activity was obtained by A. sojae ATCC 20235 and mutants thereof. The highest polygalacturonase activity $(1,087.2 \pm 151.9 \mathrm{U} / \mathrm{g})$ in solid-state culture was obtained by mutant M3, which was 1.7 times increased in comparison to the wild strain, A. sojae ATCC 20235. Additional, further mutation of mutant M3 for two more cycles of treatment by UV irradiation generated mutant DH56 with the highest polygalacturonase activity $(98.8 \pm 8.7 \mathrm{U} / \mathrm{mL})$ in submerged culture. This corresponded to 2.4-fold enhanced polygalacturonase production in comparison to the wild strain. The results of this study indicated the development of a classical mutation and selection strategy as a promising tool
\end{abstract}

D. Heerd · M. Fernández-Lahore $(\bowtie)$

Downstream Processing Laboratory, Jacobs University Bremen gGmbH, Campus Ring 1, 28759 Bremen, Germany

e-mail: m.fernandez-lahore@jacobs-university.de

C. Tari

Department of Food Engineering, İzmir Institute of Technology, Gulbahce Campus, 35430 Urla, İzmir, Turkey to improve pectinolytic enzyme production by both fungal strains.

Keywords Strain improvement · Mutagenesis · Aspergillus sojae $\cdot$ Pectinase $\cdot$ Polygalacturonase

\section{Introduction}

Nowadays, microbes are routinely used in large-scale processes of fermentation for the commercial production of enzymes such as proteases, cellulases and pectinases. Economics of such processes might be improved by overproduction of natural products, assimilation of inexpensive and complex raw materials or reduction of fermentation time. Microbial strain improvement is one of the methods used to target improvement of fermentation economics. Success in making and keeping a fermentation industry competitive depends greatly on continuous improvement of the production strain (BarriosGonzález 2012). Development of industrial strains is based on changes in the microbial DNA sequence, which is achieved by mutation, genetic recombination or genetic engineering techniques. Traditional strain improvement programs employ mutagenesis followed by screening or selection. These classical strain improvement methods have historical use. Hence, strains derived from non-recombinant methods or classical strain improvement are widely accepted as less significant process changes, assuming that product specifications are met and regulatory notification is completed (Parekh et al. 2000).

Mutagenesis is generating novel genotypes either unintentionally (spontaneous mutation) or intentionally (induced mutation). Induced mutations are achieved by subjecting the genetic material to physical or chemical agents called mutagens. Conventional mutagens employed for strain improvement include $N$-methyl- $N$ '-nitro- $N$-nitrosoguanidine (NTG), 
ethyl methanesulfonate (EMS), hydroxylamine $\left(\mathrm{NH}_{2} \mathrm{OH}\right)$, nitrous acid $\left(\mathrm{HNO}_{2}\right)$ and ultraviolet rays (UV) (Demain and Adrio 2008; Parekh et al. 2000). Each mutagen causes DNA alterations in a specific manner. A mutagen may also induce more than one type of lesion. Most mutagenic agents cause some damage to the DNA through deletion, addition, transversion or substitution of bases or breakage of DNA strands. For example, mutation by UV irradiation induces pyrimidine dimerization and cross-links in DNA (Parekh et al. 2000).

A common method used is treating cells with a mutagen until a certain "desired" kill is obtained. Often high doses of mutagen are applied to kill most microorganisms in order to increase frequency of generated mutants (Demain and Adrio 2008; Meireles et al. 2002; Smith and Wood 1991). However, the optimum dose of mutagen is that which gives the highest proportion of desirable mutants in the surviving population. There is no simple linear relationship between frequency of mutants and mutagen dosage or survival rate. It was even reported that low doses of mutagen (20-50\% survival) yielded in highest mutant frequency for different types of mutants. Furthermore, high mutagen doses can produce chromosome rearrangements and in general disturb the genetic background by an enhanced load of undesirable mutations especially utilizing recurrent mutagenic treatment (Bos 1987).

Besides modifying a strain, the identification and selection of improved mutants is an important part in strain improvement. Screening strategies may be divided into two basic types: (a) the non-selective random screening, where randomly picked isolates are tested for desired qualities; and (b) rationalized selection, based on some form of pre-selection (Parekh et al. 2000).

Although the classical strain engineering approach is quite old and seems to be labour intensive, it is having a record of success of remarkable enhanced product yields through the use of mutagenesis and screening, e.g. the classical example is the significant increased penicillin production by Penicillium chrysogenum (Sarkar and Saunders 1999). Moreover, the developments in high-throughput screening and analytical technologies enable quick screening and evaluation of large mutant libraries under various process-like conditions. However, the induced changes via the classical strain engineering approach are not easily traceable or movable to another host strain, and the organisms used for high production of a desired compound are often genetically uncharacterized. Thus, this approach can be generally applied to any strain of interest, which includes intensively studied model organisms and newly isolated species as well. On the contrary, the newer approaches of recombinant DNA technology enable targeted mutagenesis to create desired phenotypes. However, without fundamental knowledge of the organism, it is impossible to approach genetic engineering via recombinant DNA techniques in a rational manner. Importantly, in contrast to newer recombinant DNA-based technologies, strains developed via classic methods are not considered as genetically modified organism (GMO), which removes significant barriers to their acceptance by regulatory agencies and also by consumer. The classical approach of phenotype optimization is well established in the food industry due to "generally recognized as safe" (GRAS) classification and ease of selection (Crook and Alper 2012; Patnaik 2008).

Classical strain development approaches have been also successful applied for improved carbohydrate-active enzyme production by fungi (Smith and Wood 1991; Antier et al. 1993; Hadj-Taieb et al. 2002; Adsul et al. 2007). Filamentous fungi are the major producers of pectinolytic enzymes, such as polygalacturonase $(\mathrm{PG})$, which are extensively used in food industry, e.g. for the extraction and clarification of fruit juices or in winemaking (Kashyap et al. 2001; Naidu and Panda 1998). Depolymerizing enzymes like PGs are distinguished according to their substrate preference, whether they have preference for poly $[\alpha(1 \rightarrow 4)$-D-methylgalacturonic acid] (pectin-like substrates), which are termed as PMG in this study or poly $[\alpha(1 \rightarrow 4)$-D-galacturonic acid] (pectic acid-like substrates), which are termed as PG (Whitaker 1984). Furthermore, these enzymes are termed as exo- or endo-enzymes depending on the action pattern. Endo-PGs randomly attack the $[1 \rightarrow 4] \alpha$-glycosidic linkages of the polysaccharide chain producing a number of galacturonic acid oligomers, while exo-PGs specifically hydrolyses at the non-reducing end of polygalacturonic acid. Commercial pectic enzymes used in food industry normally contain a mixture of enzymes that split pectic compounds; traditionally mixtures consist of PG, pectin lyase (PL) and pectin methylesterase (PME), and are associated with cellulolytic, proteolytic and other species of enzymes apart from the main pectinases (Del Cañizo et al. 1994). Aspergillus species produce a large number of enzymes particularly involved in the degradation of pectic substances (van den Brink and de Vries 2011). The composition of the enzyme set differs significantly between the fungal species (Benoit et al. 2012). The fungal biodiversity with respect to plant cell wall degradation, including the degradation of pectic substances, has industrial importance for utilization of desired enzyme sets as needed for certain applications. In some food processes, it is convenient to use only one type of pectinolytic enzymes, e.g. preparation of instant potato flakes and carrot juice for baby food requires the maceration, where vitamins, colour and aroma have to be preserved and for these applications preparations that mainly contain PG activity are preferred (Lang and Dörnenburg 2000).

Previous studies demonstrated already the potential of pectinase production by Aspergillus sojae in both fermentation systems (Gögus et al. 2006; Heerd et al. 2012; Ustok et al. 2007). Moreover, providing selected PG overproducing mutants, which were generated by the work described herein, for fermentation process parameter optimization studies focusing 
on PG titers, demonstrated the successful process development in submerged or solid-state culture (Buyukkileci et al. 2011; Demir et al. 2012).

The work reported herein discusses the use of the classical strain engineering approach for enhanced PG production by A. sojae in submerged fermentation $(\mathrm{SmF})$ and solid-state fermentation (SSF) applying an optimized mutation and screening methodology. Furthermore, the results of this study provide a comparison of pectinolytic enzyme sets obtained in SmF and SSF, which were characterized on the basis of their substrate degrading mode.

\section{Materials and methods}

\section{Materials}

All chemicals were purchased from AppliChem GmbH (Darmstadt, Germany), except substrates for detection of pectinolytic activities, e.g. polygalacturonic acid, polygalacturonic acid sodium salt and pectin, and mutagen NTG were obtained from Sigma-Aldrich Chemie GmbH (Steinheim, Germany). NTG stock solution was prepared by dissolving in phosphate-buffered saline (PBS) buffer and sterile filtration through a $0.2-\mu \mathrm{m}$ syringe filter. The solution was stored in aliquots in dark sterile tubes at $-20^{\circ} \mathrm{C}$.

Microbial substrates like wheat bran, sugar beet pulp pellets and molasses were obtained from local suppliers (Bremer Rolandmühle Erling GmbH \& Co. KG, Bremen, Germany; Nordzucker AG, Uelzen, Germany; Golden Sweet, Meckenheim, Germany).

\section{Microorganisms}

Fungal strains of $A$. sojae ATCC 20235, purchased from Procochem Inc (Teddington, United Kingdom), and A. sojae CBS 100928, obtained from the Centraalbureau voor Schimmelcultures (CBS) (Utrecht, Netherlands), were propagated on agar plates according to the specifications given in Heerd et al. (2012). It has to be noted that $A$. sojae ATCC 20235, which is still deposited as $A$. sojae at the ATCC, did not meet the requirements to be classified as $A$. sojae on the basis of morphological parameters (Ushijima et al. 1982) and has been reclassified as Aspergillus oryzae based on the alpA restriction fragment length polymorphism (RFLP) (Heerikhuisen et al. 2005).

The spore suspensions used for mutagenesis and as inoculum for submerged and solid-state cultures were obtained from molasses agar slants containing the following: glycerol $(45 \mathrm{~g} / \mathrm{L})$, molasses $(45 \mathrm{~g} / \mathrm{L})$, peptone $(18 \mathrm{~g} / \mathrm{L}), \mathrm{NaCl}(5 \mathrm{~g} / \mathrm{L})$, $\mathrm{KCl}(0.5 \mathrm{~g} / \mathrm{L}), \mathrm{FeSO}_{4} \cdot 7 \mathrm{H}_{2} \mathrm{O}(15 \mathrm{mg} / \mathrm{L}), \mathrm{KH}_{2} \mathrm{PO}_{4}(60 \mathrm{mg} / \mathrm{L})$, $\mathrm{MgSO}_{4}(50 \mathrm{mg} / \mathrm{L}), \mathrm{CuSO}_{4} \cdot 5 \mathrm{H}_{2} \mathrm{O}(12 \mathrm{mg} / \mathrm{L}), \mathrm{MnSO}_{4} \cdot \mathrm{H}_{2} \mathrm{O}$ $(15 \mathrm{mg} / \mathrm{L})$ and agar $(20 \mathrm{~g} / \mathrm{L})$. High spore production was achieved by cultivation on molasses agar slants at $30{ }^{\circ} \mathrm{C}$ for 1 week.

Screening medium

Screening medium was used for the selection of pectinase overproducing mutants of $A$. sojae. The medium was a modification of the selection medium described by Durrands and Cooper (1988). Two hundred fifty millilitres of $0.1 \mathrm{M}$ acetate buffer (pH 5.0) containing $1 \mathrm{~g} \mathrm{NaNO}_{3}, 0.5 \mathrm{~g} \mathrm{KH}_{2} \mathrm{PO}_{4}, 0.25 \mathrm{~g}$ $\mathrm{MgSO}_{4} \cdot 7 \mathrm{H}_{2} \mathrm{O}$ and $7.5 \mathrm{~g}$ agar was sterilized by autoclaving. Warm solution was blended with equal volume of separately sterilized solution of $0.5 \%(w / v)$ polygalacturonic acid sodium salt and poured into Petri dishes. Pectinase activity was detected as clear zone around colonies in the background of the precipitated substrate after treating plates with $1 \%(w / v)$ cetyltrimethyl ammonium bromide solution.

\section{Strain improvement}

The spore suspension $\left(10^{7}\right.$ spores $/ \mathrm{mL}$ in PBS buffer, $\mathrm{pH}$ 7.4) of $A$. sojae was treated with ultraviolet (UV) radiation and/or NTG to obtain PG hyperproducing mutants. Treated samples were suitably plated on yeast malt extract (YME) plates and incubated at $30^{\circ} \mathrm{C}$ for $48 \mathrm{~h}$. The colony forming unit was used to calculate the survival rate. Colonies were further incubated at $30{ }^{\circ} \mathrm{C}$ and mutants were morphologically selected for the presence of sporulation. The isolation of mutants consisted in the isolation of colonies originated from single conidia. Mutants obtained were further selected by replica-plating on screening medium. The selection of "zone mutants" based upon the observation of an enhanced zone of hydrolysis. Mutants were confirmed for increased PG production in SSF. The mutant strain producing the highest PG activity was selected for further mutation treatment.

Three strategies of mutagenesis were employed for strain improvement of $A$. sojae (Fig. 1): repeated treatment of spores by UV irradiation (a), repeated treatment by NTG (b) and sequential treatment by combination of NTG and UV irradiation (c). In all cases, mutants were generated in 3 cycles of mutation; however, mutant M3 descending from A. sojae ATCC 20235 was treated by UV radiation for two more cycles which generated mutant DH56. All generated mutants and the method of their generation are presented in Fig. 1.

\section{UV mutagenesis}

Mutagenesis was performed using a modification of the method given by De Nicolás-Santiago et al. (2006) under semidarkened conditions, transmitting UV light in a box and mutagenic treatment of the spore suspension was done under agitation using a magnetic bar. Exposure time to UV radiation under the described conditions was previously explored and 
Fig. 1 Scheme of repeated and sequential mutagenesis

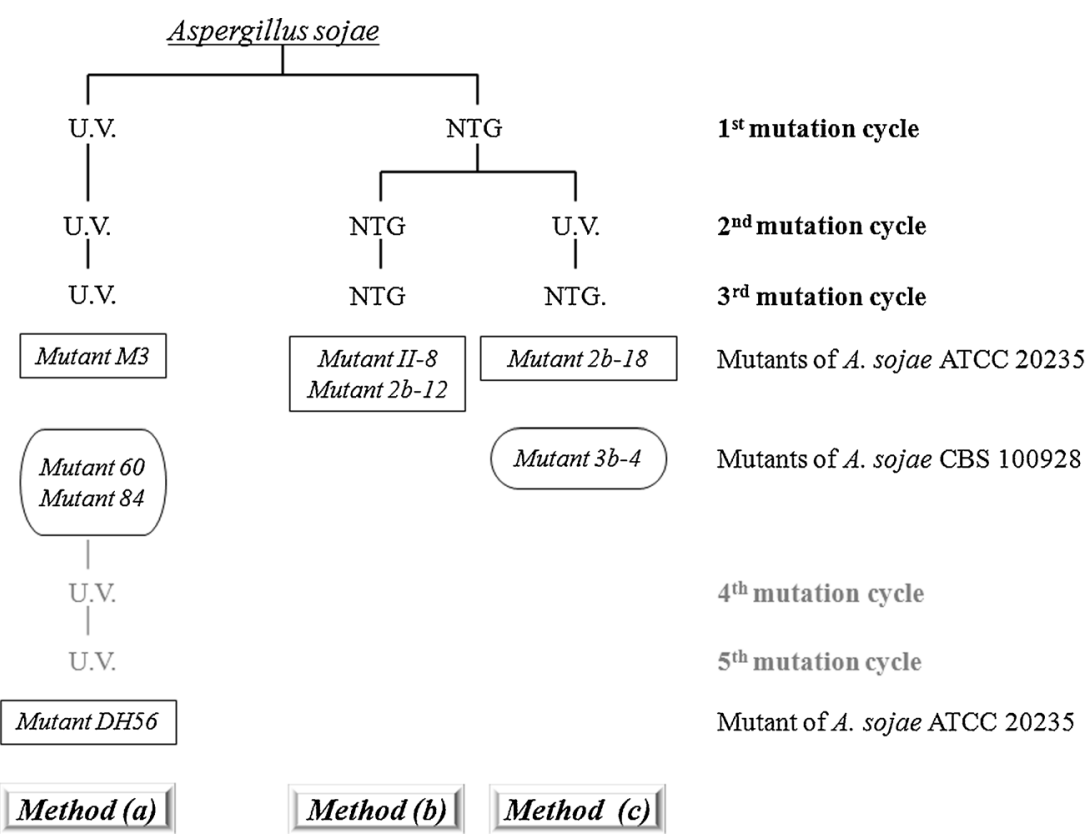

repetitions resulted in high variations of survival rates (data not shown). Nevertheless, $5 \mathrm{~min}$ exposure time with placement of the 254-nm-wavelength germicidal lamp $17 \mathrm{~cm}$ above the spore suspension caused adequate inactivation of spores from both fungal strains. Treated samples were placed on ice in the dark for $5 \mathrm{~min}$, followed by $30 \mathrm{~min}$ incubation in darkness at room temperature to avoid photoreactivation repair. Suitable plating and isolation of mutants was done as aforementioned.

\section{Mutagenesis by NTG}

Treatment of spores by NTG was performed in dark Eppendorf test tubes at $600 \mathrm{rpm}$. The procedure for treatment with NTG was optimized applying statistical experimental design to obtain a survival rate in the range of 50-60\%. In an initial screening experiment, the effect of three factors (NTG concentration, incubation time and temperature) on the survival rate was investigated using fractional factorial design (Table 1). The design matrix consisted of 14 experimental runs including a replicate and three centre points. The factor settings were finally optimized for mutagenesis of each fungal strain (Table 2). The optimization experimental design comprised 34 experiments for each strain including a replicate and three centre points using central composite face-centered (CCF) design. Experiments were evaluated using Multiple Linear Regression (MLR) utilizing the MODDE 9.0 software package, supplied by Umetrics AB, Umeå, Sweden. Finally, validation experiments were performed in triplicate to confirm optimal factor settings (Table 3 ).
Solid-state fermentation and enzyme leaching

SSF was performed in culture flasks $(250 \mathrm{~mL})$ utilizing $10 \mathrm{~g}$ of wheat bran and ground sugar beet pellets in the ratio 70:30, wetted at $160 \%$ with $0.2 \mathrm{~N} \mathrm{HCl}$ solution (sterilized at $121{ }^{\circ} \mathrm{C}$ for $20 \mathrm{~min}$ ). The mentioned moisture level was calculated as dry basis moisture content according to the following equation:

Moisture content $(\%)=\left(\right.$ weight $_{\text {wet }}-$ weight $\left._{\text {dry }}\right) /$ weight $_{\text {dry }} \times 100$

where weight $_{\text {wet }}$ is the weight of solid medium together with

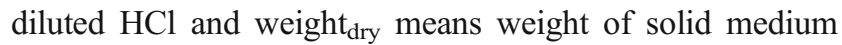
(natural moisture content in the solid medium components has not been considered for calculation).

The inoculum was adjusted to $1 \times 10^{6}$ spores per gram dry weight, which corresponds to $3.8 \times 10^{5}$ spores per gram moist mass of medium. Flasks were incubated at $30{ }^{\circ} \mathrm{C}$ for 8 days and shaken manually twice at the day of inoculation and the first cultivation day.

Enzyme leaching was performed with $80 \mathrm{~mL}$ tap water for $20 \mathrm{~min}$ at $250 \mathrm{rpm}$ agitation and $25^{\circ} \mathrm{C}$. Mycelium and solid medium were separated by centrifugation at $4{ }^{\circ} \mathrm{C}$ and $3,220 \times g$ for $20 \mathrm{~min}$. Enzyme activities and total protein content were determined in the supernatant. 
Table 1 Screening factors and responses in NTG treatment applying fractional factorial design

\begin{tabular}{|c|c|c|c|c|c|}
\hline \multirow[t]{2}{*}{$\begin{array}{l}\text { Exp. } \\
\text { no. }\end{array}$} & \multicolumn{3}{|l|}{ Factors } & \multicolumn{2}{|c|}{$\begin{array}{l}\text { Response: survival } \\
\operatorname{rate}^{\mathrm{a}}(\%)\end{array}$} \\
\hline & $\begin{array}{l}\text { NTG } \\
(\%)\end{array}$ & $\begin{array}{l}\text { Time } \\
\text { (h) }\end{array}$ & $\begin{array}{l}\text { Temperature } \\
\left({ }^{\circ} \mathrm{C}\right)\end{array}$ & $\begin{array}{l}\text { A. sojae } \\
\text { A T C C } \\
200235\end{array}$ & $\begin{array}{l}\text { A. sojae } \\
\text { C B S } \\
100928\end{array}$ \\
\hline 1 & 0.005 & 0.5 & 30 & 100 & 60 \\
\hline 2 & 0.05 & 0.5 & 16 & 46 & 44 \\
\hline 3 & 0.005 & 3 & 16 & 89 & 60 \\
\hline 4 & 0.05 & 3 & 30 & 16 & 2 \\
\hline 5 & 0.0275 & 1.75 & 23 & 64 & 44 \\
\hline 6 & 0.0275 & 1.75 & 23 & 73 & 44 \\
\hline 7 & 0.0275 & 1.75 & 23 & 70 & 39 \\
\hline 8 & 0.005 & 0.5 & 30 & 100 & 62 \\
\hline 9 & 0.05 & 0.5 & 16 & 66 & 36 \\
\hline 10 & 0.005 & 3 & 16 & 100 & 85 \\
\hline 11 & 0.05 & 3 & 30 & 10 & 3 \\
\hline $12-14^{\mathrm{b}}$ & 0.0275 & 1.75 & 23 & $72.7 \pm 4.7$ & $41.0 \pm 2.0$ \\
\hline
\end{tabular}

${ }^{a}$ The observed values of survival rate were the mean values calculated from duplicates of colony counts

${ }^{\mathrm{b}}$ Three repetitions of the center point gave a standard variation below $5 \%$

\section{Submerged fermentation}

The composition of $\mathrm{SmF}$ medium comprised $95 \mathrm{~g} / \mathrm{L}$ molasses, $10 \mathrm{~g} / \mathrm{L}\left(\mathrm{NH}_{4}\right)_{2} \mathrm{SO}_{4}$ and $30 \mathrm{~g} / \mathrm{L}$ sugar beet pellets. The $\mathrm{pH}$ was adjusted to 4.0 before sterilization $\left(121{ }^{\circ} \mathrm{C}\right.$ for $\left.20 \mathrm{~min}\right)$. Fermentation was carried out in 250 -mL Erlenmeyer flasks containing $30 \mathrm{~mL}$ of medium at $30{ }^{\circ} \mathrm{C}$ and $250 \mathrm{rpm}$ for 4 days. Flasks were inoculated with $3.8 \times 10^{5}$ spores per $\mathrm{mL}$ of medium.

Enzyme activities and total protein content were determined in the supernatant obtained after centrifugation of the fermentation broth at $4{ }^{\circ} \mathrm{C}$ and $3,220 \times g$ for $20 \mathrm{~min}$.

Table 2 Selected variables and their assigned levels by CCF

\begin{tabular}{lllll}
\hline Microorganism & Factor (unit) & \multicolumn{3}{l}{ Actual factor levels } \\
\cline { 3 - 5 } & & -1 & 0 & +1 \\
\hline \multirow{2}{*}{ A. sojae ATCC 20235 } & NTG concentration (\%) & 0.01 & 0.055 & 0.1 \\
& Incubation time (h) & 0.5 & 1.75 & 3 \\
& Temperature $\left({ }^{\circ} \mathrm{C}\right)$ & 15 & 25 & 35 \\
A. sojae CBS 100928 & NTG concentration (\%) & 0.005 & 0.0275 & 0.05 \\
& Incubation time (h) & 0.2 & 1.6 & 3 \\
& Temperature $\left({ }^{\circ} \mathrm{C}\right)$ & 14 & 22 & 30 \\
\hline
\end{tabular}

Exo-pectinolytic activity measurement

\section{Polygalacturonase assay}

Exo-PG activity was assayed according to the procedure provided by Panda et al. (1999) with slight modifications. In brief, samples of $0.086 \mathrm{~mL}$ containing appropriate diluted PG enzyme were mixed with $0.4 \mathrm{~mL}$ of $2.4 \mathrm{~g} / \mathrm{L}$ polygalacturonic acid solution dissolved in $0.1 \mathrm{M}$ acetate buffer ( $\mathrm{pH}$ 4.8). This mixture was incubated at $40{ }^{\circ} \mathrm{C}$ for $10 \mathrm{~min}$. The reducing sugar released was measured by using the Nelson-Somogyi method (Nelson 1944) as adapted by Panda et al. (1999). First reaction was terminated by adding $0.5 \mathrm{~mL}$ copper reagent and placing the mixture in boiling water for $10 \mathrm{~min}$. After cooling down, $1 \mathrm{~mL}$ of arsenomolybdate reagent was added, followed by intensive vortexing and centrifugation at $3,220 \times g$ at $22{ }^{\circ} \mathrm{C}$ for $5 \mathrm{~min}$. The absorbance of the supernatant was read on a spectrophotometer at $500 \mathrm{~nm}$. Blanks were in-cooperated containing all the reagents and the enzyme, but the enzyme was not allowed to react with the substrate. Standard solutions of galacturonic acid were used for calibration. One unit of exo-PG activity was defined as the amount of enzyme that catalyzes the release of $1 \mu \mathrm{mol}$ of galacturonic acid per unit volume of sample per minute under the standard assay conditions mentioned above.

\section{Polymethylgalacturonase assay}

Exo-PMG activity was determined according to the method provided by Panda et al. (1999) with slight modifications, using $0.5 \mathrm{~g} / \mathrm{L}$ pectin as substrate dissolved in $0.1 \mathrm{M}$ acetate buffer ( $\mathrm{pH} 5.0)$. Crude extract $(0.086 \mathrm{~mL}$ ) containing enzyme was added to $0.4 \mathrm{~mL}$ substrate and incubated for $10 \mathrm{~min}$ at $40{ }^{\circ} \mathrm{C}$. The reducing sugar released was measured using the Nelson-Somogyi method (Nelson 1944) as adapted by Panda et al. (1999), and as described for the PG assay. Standard solutions of galacturonic acid were used for calibration. One unit of exo-PMG activity was defined as the amount of enzyme that catalyzes the release of $1 \mu \mathrm{mol}$ of galacturonic acid per unit volume of supernatant per minute under standard assay conditions mentioned above.

Endo-pectinolytic activity measurement

Endo-enzyme activities were determined by measuring the decrease in viscosity of a substrate solution, either $2 \%(w / v)$ pectin for endo-PMG or $3.2 \%(w / v)$ polygalacturonic acid (sodium salt) for endo-PG. Reduction in viscosity was determined according to a modified method of Mill and Tuttobello (1961), utilizing a graduated glass pipette as viscometer.

Pectinolytic activity was assayed by adding $0.2 \mathrm{~mL}$ of an appropriate diluted enzyme sample, to $0.2 \mathrm{~mL}$ of $0.2 \mathrm{M}$ acetate buffer (pH 5.0) and $1.6 \mathrm{~mL}$ substrate. The mixture was 
Table 3 Validation experiments and results at optimized factor settings for desired survival rates of $A$. sojae

The observed values of survival rate were the mean values with standard deviation (mean $\pm \mathrm{SD}$ ) calculated from triplicates of colony counts

\begin{tabular}{llllll}
\hline Microorganism & Run & $\begin{array}{l}\text { NTG concentration } \\
(\%)\end{array}$ & $\begin{array}{l}\text { Temperature } \\
\left({ }^{\circ} \mathrm{C}\right)\end{array}$ & $\begin{array}{l}\text { Incubation time } \\
(\mathrm{h})\end{array}$ & $\begin{array}{l}\text { Survival rate }^{\mathrm{a}} \\
(\%)\end{array}$ \\
\hline $\begin{array}{lllll}\text { A. sojae ATCC } \\
20235\end{array}$ & I & 0.1 & 20 & 1 & $64 \pm 7$ \\
A. sojae CBS & II & 0.1 & 20 & 1.25 & $52 \pm 4$ \\
100928 & I & 0.04 & 30 & 0.7 & $37 \pm 3$ \\
& II & 0.03 & 22 & 1.6 & $63 \pm 3$ \\
& III & 0.05 & 14 & 0.76 & $67 \pm 1$ \\
& IV & 0.04 & 25 & 0.7 & $49 \pm 1$ \\
& V & 0.03 & 30 & 0.8 & $48 \pm 10$ \\
& VI & 0.05 & 25 & 0.6 & $46 \pm 4$ \\
\hline
\end{tabular}

incubated in a water bath for $1 \mathrm{~h}$ at $40^{\circ} \mathrm{C}$. After incubation, the mixture was cooled down for $30 \mathrm{~s}$ in ice-cold water and viscosity of the samples was determined. The later was done indirectly by measuring the time required for $0.9 \mathrm{~mL}$ of reaction mixture to elute through a $1.0-\mathrm{mL}$ glass pipette. Viscosity was calculated from a calibration curve obtained by time measurements to pass polyvinylpyrrolidone (PVP) 360 aqueous standard solutions through the pipette at $25^{\circ} \mathrm{C}$, which were previously passed through an Ostwald viscometer at $25{ }^{\circ} \mathrm{C}$. Controls for non-enzymatically treated substrate solutions were included, utilizing $0.2 \mathrm{~mL}$ water instead of enzyme samples. One unit of endo-pectinase (either PG or PMG) activity was defined according to Patil and Dayanand (2006), as the quantity of enzyme which caused a $50 \%$ reduction in viscosity of the reaction mixture per minute, under the assay conditions described above.

\section{Total soluble protein measurement}

Total extracellular protein was measured according to the modified Bradford method (Bradford 1976), using Coomassie Plus ${ }^{\text {TM }}$ Protein Assay Kit (Pierce, Fischer scientific, Schwerte, Germany). The assay was performed in a microplate by determining the absorbance at $595 \mathrm{~nm}$ using bovine serum albumin (BSA) as a standard.

\section{Results}

\section{Optimization of mutagenesis by NTG}

Optimization of the NTG treatment targeted the achievement of a desired survival rate of 50-60\% by mutagenesis. It was performed in two steps, comprising a screening of three factors and optimization of their settings. In the first screening step, the effect of NTG concentration $(0.005-0.05 \%)$, incubation time $(0.5-3 \mathrm{~h})$ and temperature $\left(16-30{ }^{\circ} \mathrm{C}\right)$ on the survival rate was studied (Table 1). Evaluation of the experimental data identified a sound model quality for the survival rate of spores from $A$. sojae ATCC $20235\left(R^{2} / Q^{2} 0.95 / 0.88\right)$ as well as for spores of $A$. sojae CBS $100928\left(R^{2} / Q^{2} 0.94 / 0.80\right)$, and the linear models showed no lack of fit (A. sojae ATCC 20235: $p=0.205$; . sojae CBS 100928: $p=0.588$ ). All three investigated factors had significant effect on the survival rate of spores from both fungal strains and the desired survival rate of 50-60\% was achieved for both strains in the chosen factor range (Fig. 2). However, spores of $A$. sojae ATCC 20235 seemed to be more resistant to the mutagenic treatment comparing the survival rates of both strains under the same conditions. Hence, to obtain the desired survival rate by $A$. sojae ATCC 20235 a higher NTG concentration, longer incubation time or higher incubation temperature was needed. Moreover, there was a stronger influence of temperature for the mutagenesis of $A$. sojae CBS 100928. Therefore, factor settings were optimized at different ranges for both strains and the temperature range was decreased for the optimization step applying $A$. sojae CBS 100928 (Table 2). Furthermore, NTG concentration had to be increased for the optimization experiments at lower temperature to get a survival rate of 50-60\% for $A$. sojae ATCC 20235. Evaluation of the optimization results from $A$. sojae ATCC 20235, utilizing the optimizer tool of MODDE 9.0, resulted in a clear identification of the factor settings for NTG concentration and temperature with $0.1 \%$ and $20^{\circ} \mathrm{C}$, respectively. Therefore, these factors were fixed and validation experiments focused on incubation time testing the suggested value of 1 and $1.25 \mathrm{~h}$ (Table 3 ). Validation experiments identified $1.25 \mathrm{~h}$ incubation time at $0.1 \%$ NTG concentration and $20{ }^{\circ} \mathrm{C}$ as optimal setting to achieve a survival rate of $50-60 \%$ by mutagenesis of $A$. sojae ATCC 20235.

Evaluation of data from $A$. sojae CBS 100928 did not result in a clear identification of factor settings. The desired survival rate of 50-60\% could be obtained at all applied temperature levels using diverse factor settings for NTG concentration and incubation time (Fig. 3). The lower the temperature, the more harsh conditions for NTG concentration and longer incubation time is needed to achieve the desired survival rate. Therefore, validation experiments were performed at broad factor ranges (Table 3, run I-III). Since the desired survival rate of 50-60\% was not obtained under these conditions and the strong 
Fig. 2 Sweet spot plot of desired survival rate in screening experiments of a $A$. sojae ATCC 20235 and b $A$. sojae CBS 100928. The green zone represents the conditions yielding a survival rate of $50-60 \%$ a

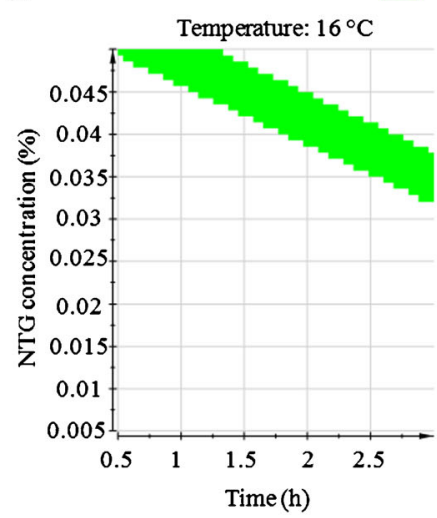

b

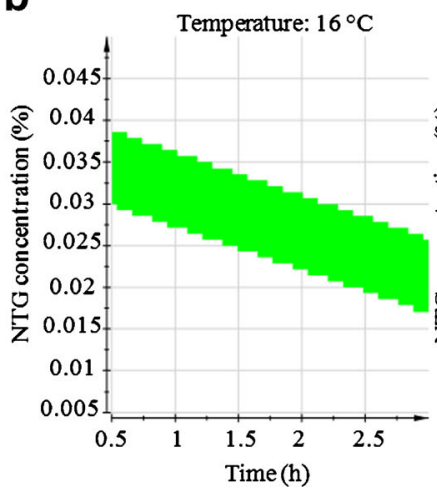

Sweet spot $=50-60 \%$ survival rate
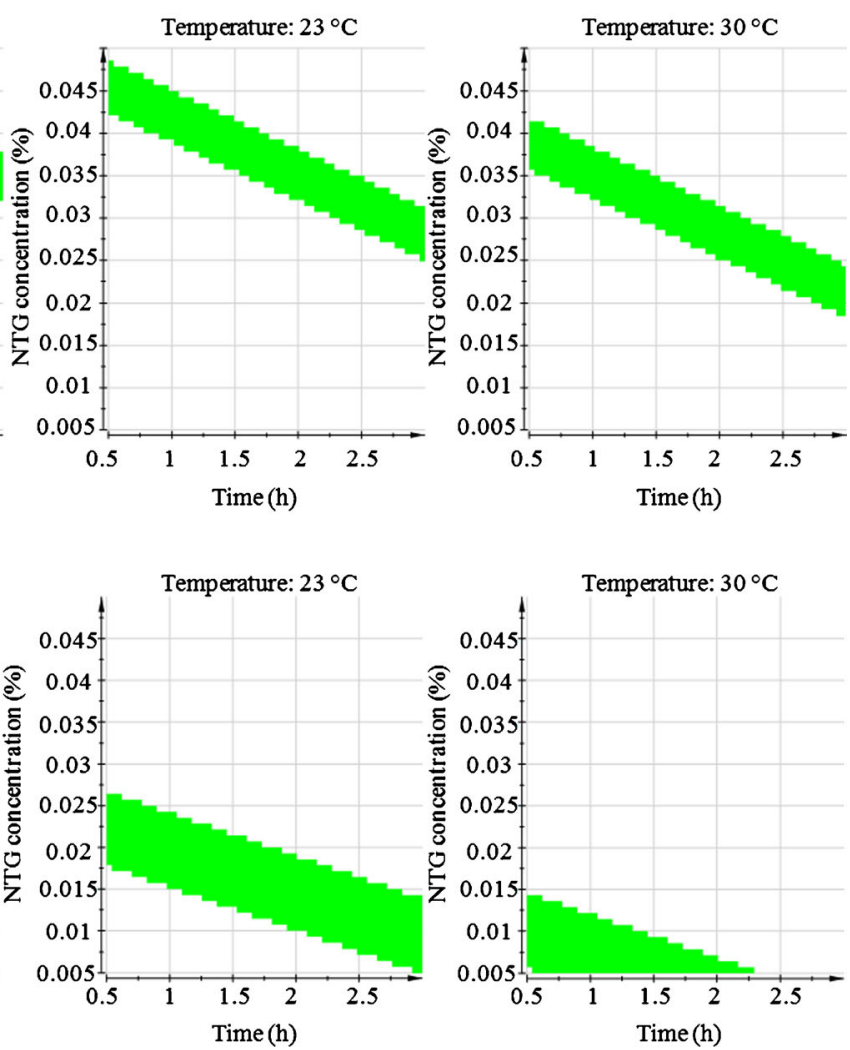

influence of temperature on the survival rate of $A$. sojae CBS 100928 was known from previous screening experiments, further validations experiments were added with increased temperature and adapted incubation times (Table 3, run IVVI). The factor settings of run IV resulted in almost $50 \%$ survival rate. Hence, procedure of mutagenesis of $A$. sojae CBS 100928 was fixed at $0.04 \%$ NTG concentration at $25^{\circ} \mathrm{C}$ for $0.7 \mathrm{~h}$.

Fig. 3 Contour plots of the survival rate obtained by NTG treatment of A. sojae CBS 100928
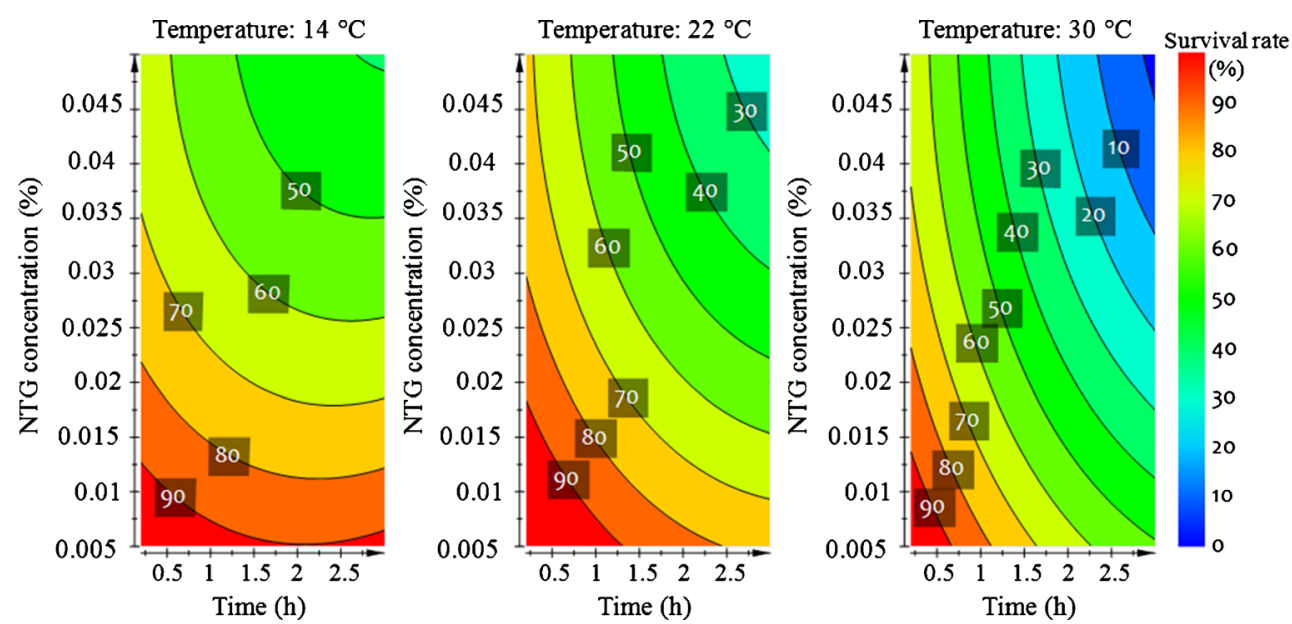

\section{Comparison of exo-pectinolytic enzyme production in SSF}

Pectinase production was investigated on solid medium containing sugar beet pulp as inducer substrate which was previously optimized for enhanced PG production by $A$. sojae. The highest PG activity of $1,087.2 \pm 151.9 \mathrm{U} / \mathrm{g}$ in solid-state culture was obtained by mutant $\mathrm{M} 3$, descending from $A$. sojae ATCC 20235 (Table 4). This corresponded to a 1.7-fold 


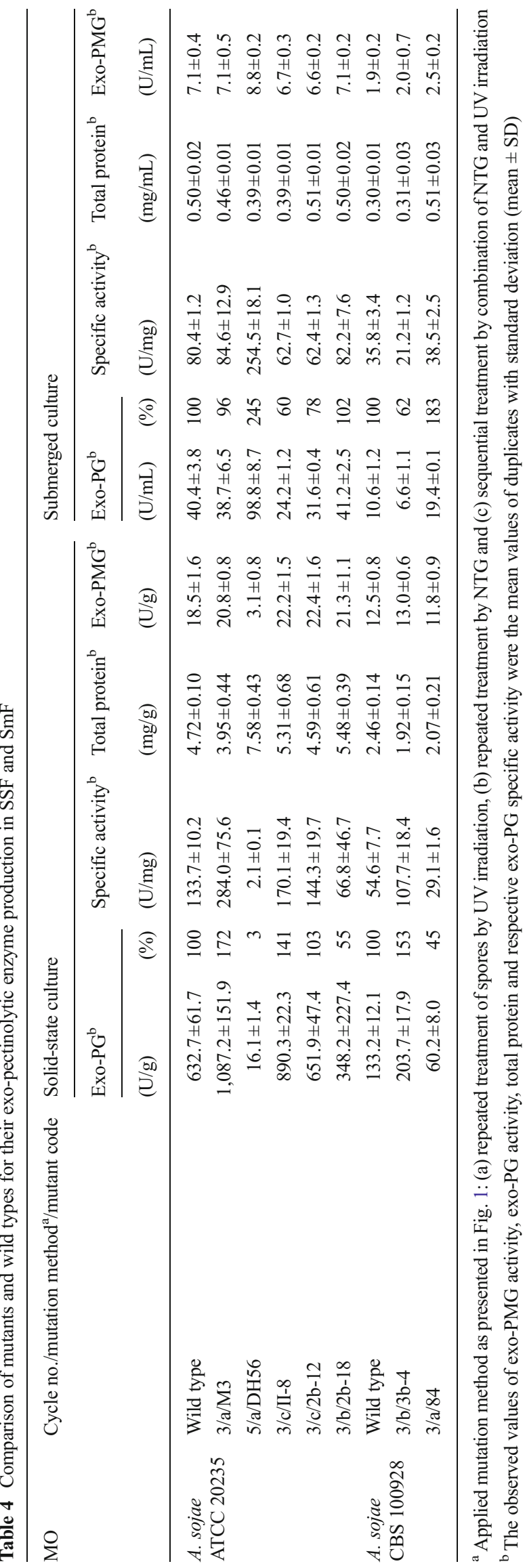

increase of enzyme activity obtained after 3 cycles of UV irradiation. Regarding PG activity, mutant M3 produced also the highest specific activity in SSF, which was 2.1-fold increased compared to the wild strain. Mutation by UV irradiation slightly enhanced exo-PMG activity by $12 \%$, too.

Further UV treatment of this mutant by two more cycles generated mutant DH56 (Fig. 1, method a) with increased pectinase activity on screening medium agar plate (data not shown), but significantly decreased PG activity in SSF. The high protein content produced by this mutant in SSF is conspicuous in contrast to the low PG activity. Exo-enzyme activity degrading pectin is also low compared to the wild strain, which indicated that more proteins, which are not involved in the degradation of pectic substances, were secreted by mutant DH56 in SSF.

The high standard deviation indicated that results produced by mutant $2 \mathrm{~b}-18$, which was generated by 3 cycles of treatment with NTG (Fig. 1, method b), were not reproducible. Furthermore, PG activity decreased by each new generation cycle, indicating that the generated mutant was not stable. Hence, mutant $2 b-18$ was excluded from further considerations.

Increased pectinolytic activities of mutants generated by a combination of NTG treatment and UV irradiation (Fig. 1, method c) were stable over repeated generation cycles. Mutant II-8 showed 1.4-fold increased PG activity in SSF, while mutant 2b-12 achieved a similar PG activity in SSF like the wild strain. Their specific PG activities were higher compared to the wild type. Looking at their exo-PMG activities, these mutants produced even higher pectin degrading activities than mutant M3. The highest exo-PMG activity in SSF was obtained by mutant $2 \mathrm{~b}-12$, which was $21 \%$ increased compared to the wild type.

Following previous findings of Heerd et al. (2012), PG production by $A$. sojae CBS 100928 was significant lower in comparison to $A$. sojae ATCC 20235 (Table 4). Considering mutants descending from $A$. sojae CBS 100928 for PG production in SSF, only mutant $3 \mathrm{~b}-4$ showed increased exo-PG and exo-PMG activity compared to the wild type. Interestingly, this mutant, generated by treating spores with NTG (Fig. 1, method b), was stable in contrast to the mutant $2 \mathrm{~b}-18$ descending from A. sojae ATCC 20235, even though the mutagenic dose was lower for that strain (Optimization of mutagenesis by NTG). Mutant 3b-4 produced $53 \%$ increased exo-PG activity compared to its wild strain. Specific PG activity produced in SSF was even two times higher.

Pectinase activity produced in SSF by the mutant of A. sojae CBS 100928 generated by treatment with UV irradiation (Fig. 1, method a) was lower compared to the wild strain.

\section{Comparison of exo-pectinolytic enzyme production in $\mathrm{SmF}$}

The applied medium for submerged culture contained also sugar beet pulp as inducer substrate to enhance pectinase production by $A$. sojae. Evaluation of mutants descending 
from A. sojae in $\mathrm{SmF}$ gave reverse results than in SSF (Table 4). Mutants generated from A. sojae ATCC 20235 after 3 cycles UV irradiation or a combination of NTG and UV treatment produced lower PG activity in SmF than the wild strain. This might be explained by the screening method used to select mutants with enhanced PG activity in SSF. Mutant DH56 was also tested in SmF due to the low PG production in SSF but simultaneous showing a high pectinase activity on screening medium on agar plates. The PG production by mutant DH56 in SmF was 2.4-fold increased compared to the wild type, while the specific PG activity increased even by factor 3.2. Besides exo-PG activity, mutant DH56 was also the only mutant of $A$. sojae ATCC 20235 producing higher exo$\mathrm{PMG}$ activity in SmF than the wild strain.

Similar like in SSF, A. sojae CBS 100928 produced also lower PG activity $(10.6 \pm 1.2 \mathrm{U} / \mathrm{mL})$ in submerged culture in comparison to $A$. sojae ATCC 20235. PG activity obtained by A. sojae ATCC 20235 was 4.8-fold higher in SSF and 3.8-fold higher in SmF. Besides PG activity, A. sojae ATCC 20235 produced also higher PMG activity in both fermentation systems. Considering mutants descending from $A$. sojae CBS 100928 , the highest exo-PG activity of $19.4 \mathrm{U} / \mathrm{mL}$ and also the highest exo-PMG activity of $2.5 \mathrm{U} / \mathrm{mL}$ was produced by mutant 84. Hence, 3 cycles of UV radiation resulted in 1.8fold increased PG activity in SmF. PG activity in SmF of mutant of 3b-4, generated by treatment with NTG, was lower compared to the wild strain.

Comparison of endo-pectinolytic enzyme activities in SSF and $\mathrm{SmF}$

Mutants with the highest exo-PG activity in SmF and SSF were further explored for their endo-pectinase production in both fermentation systems (Table 5). Mutants with increased exo-PG activity in SSF showed also increased endo-PG activity, while utilizing these mutants in $\mathrm{SmF}$ resulted in lower exo-PG and lower endo-PG activity. Pectin degrading activity in the extracts of $A$. sojae, measured as reduction in viscosity, was significantly lower than pectic acid degrading enzyme activity. The highest endo-PG and endo-PMG activity in SSF was obtained by mutant M3, while the highest endo-PG and the highest endo-PMG activity in $\mathrm{SmF}$ were produced by mutant DH56. Following the trend of exo-pectinase activities, A. sojae ATCC 20235 produced also considerably higher endo-pectinase activities than $A$. sojae CBS 100928 in SmF and SSF.

\section{Discussion}

With any microorganism, it will usually be possible to isolate phenotypes which overproduce a product by treatment with chemical or physical mutagens, but to be successful, it is necessary to optimize the mutagenic treatment (Sarkar and Saunders 1999). The present investigation to optimize mutagenesis by NTG targeted on a survival rate between 50 and $60 \%$. This range was chosen since a low mutagen dose should be considered taking the disturbance of the genetic background into account (Bos 1987). Comparing the settings for mutagenic procedure of $A$. sojae CBS 100928 to the once of $A$. sojae ATCC 20235 showed that a conspicuous lower mutagenic dose was required to obtain the same survival rate by $A$. sojae CBS 100928 . However, repeated mutagenesis by NTG treatment produced only stable mutants of $A$. sojae CBS 100928, while repeated treatment by UV irradiation was the preferential method for generation of $\mathrm{PG}$ hyperproducing mutants of $A$. sojae ATCC 20235.

Although the filamentous fungus A. sojae ATCC 20235 produced already higher polygalacturonic acid degrading exoenzyme activities than the leading fungal pectinase producer

Table 5 Endo-pectinolytic enzyme profiles obtained in SSF and SmF

\begin{tabular}{|c|c|c|c|c|c|}
\hline \multirow[t]{3}{*}{ MO } & \multirow[t]{3}{*}{ Cycle no./mutation method $/$ mutant code } & \multicolumn{2}{|c|}{ Solid-state culture } & \multicolumn{2}{|c|}{ Submerged culture } \\
\hline & & \multicolumn{2}{|c|}{ Endo-activity ${ }^{\mathrm{b}}(\mathrm{U} / \mathrm{g})$} & \multicolumn{2}{|c|}{ Endo-activity ${ }^{\mathrm{b}}(\mathrm{U} / \mathrm{mL})$} \\
\hline & & PG & PMG & PG & PMG \\
\hline \multirow[t]{3}{*}{ A. sojae ATCC 20235} & Wild type & $869.4 \pm 75.8$ & $126.6 \pm 1.3$ & $44.4 \pm 2.3$ & $7.4 \pm 0.4$ \\
\hline & 3/a/M3 & $1,029.5 \pm 33.7$ & $129.1 \pm 0.2$ & $42.7 \pm 3.8$ & $7.7 \pm 0.1$ \\
\hline & 5/a/DH56 & $2.4 \pm 0.3$ & $3.5 \pm 1.0$ & $61.4 \pm 2.1$ & $8.0 \pm 0.03$ \\
\hline \multirow[t]{3}{*}{ A. sojae CBS 100928} & Wild type & $109.1 \pm 3.4$ & $55.3 \pm 1.5$ & $10.5 \pm 0.4$ & $3.0 \pm 0.1$ \\
\hline & $3 / b / 3 b-4$ & $114.9 \pm 2.8$ & $60.0 \pm 1.8$ & $9.5 \pm 0.6$ & $2.8 \pm 0.1$ \\
\hline & $3 / a / 84$ & $75.9 \pm 8.3$ & $29.8 \pm 2.2$ & $11.1 \pm 0.1$ & $3.0 \pm 0.02$ \\
\hline
\end{tabular}

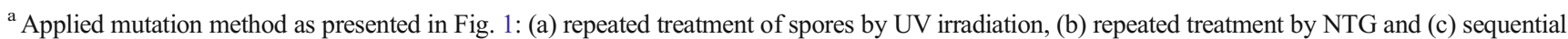
treatment by combination of NTG and UV irradiation

${ }^{\mathrm{b}}$ The observed values of endo-PG and endo-PMG activity were the mean values of triplicates with standard deviation (mean $\pm \mathrm{SD}$ )
} 
Aspergillus carbonarius (480 U/g) in SSF (Jacob 2009), the present investigation demonstrated an increase of enzyme yields for commercial considerations of PG production by A. sojae. Development and implementation of a mutation and selection strategy for the improved production of extracellular pectic acid degrading enzymes resulted in enhanced pectinolytic activities of $A$. sojae mutants. The pre-selection focused on morphological aspects regarding sporulation. Hence, generation of mutants producing sufficient amount of spores for inoculation was assured. Selection of "zone mutants" in the second step of the screening procedure enabled the detection of desired mutants with enhanced pectinase activity measured as clear zones on screening medium. Utilization of polygalacturonic acid (sodium salt) in the screening medium preferred identification of mutants with increased PG activity. Exo- and endo-pectinolytic activities degrading polygalacturonic acid were much higher than those acting on pectin (Tables 4 and 5).

Furthermore, screening on zone mutants did not distinguish between increased enzyme production in SmF or SSF system. Thus, mutants with increased PG activity either on solid substrate or in submerged culture systems were generated, meaning that mutants with increased PG production in SSF produced lower enzyme activity in $\mathrm{SmF}$ and vice versa. These findings support the information given by Barrios-González (2012) that enzyme or secondary metabolites overproducing strains, generated for SmF, generally do not perform well in SSF, and very seldom are strains efficient in both systems. Furthermore, it has been found that protein production is controlled in response to solid- or liquid-culture conditions (Barrios-González 2012). Hence, overproducing strains have to be generated particularly for the respective fermentation system. Therefore, the third screening step was focused on selection of mutants with increased PG production in SSF and only the mutants obtained after the third mutation cycle were also tested for enzyme production in SmF. Even though only mutants with increased PG production in SSF were selected for further mutagenesis, the results of this study revealed also the generation of mutants during the third mutation cycle with decreased PG production in SSF but enhanced enzyme production in SmF.

Previous comparisons of pectinase production by $A$. sojae ATCC 20235 and A. sojae CBS 100928 in SSF demonstrated already the higher enzyme production by $A$. sojae ATCC 20235 (Heerd et al. 2012). According to the present results, A. sojae ATCC 20235 produced also higher pectinase activity in submerged culture. Nevertheless, using sugar beet pulp as inducer substrate in $\mathrm{SmF}$ resulted in higher PG yields by A. sojae CBS 100928 in comparison to pectinase production by Aspergillus sp. under optimized conditions utilizing sugar beet as sole carbon source (Galiotou-Panayotou et al. 1997).

Providing the generated PG overproducing mutants for SmF process optimization studies performed by Buyukkileci et al. (2011) yielded a PG activity of $2 \mathrm{U} / \mathrm{mL}$ by $A$. sojae ATCC $20235,50.1 \mathrm{U} / \mathrm{mL}$ by mutant M3 and $98.6 \mathrm{U} / \mathrm{mL}$ by mutant DH56 (which was called mutant M5/6). In contrast to these findings, the wild strain $A$. sojae ATCC 20235 produced significant higher PG activity utilizing the economical medium described in the present study. Regarding mutant DH56, similar PG activity was obtained. Hence, the applied medium formulation seemed to be promising for optimization studies utilizing $A$. sojae for pectinolytic enzyme production in $\mathrm{SmF}$. Moreover, the high specific activity obtained by mutant DH56 in $\mathrm{SmF}$ indicated the outstanding production of PG enzyme under these cultivation conditions, which might be interesting with regard to pure enzyme formulations.

Regarding PG production in SSF, mutant M3 was characterized as potential production organism with high enzyme yields, which is promising for further optimization and scaleup studies.

In this current investigation, the use of classical strain improvement methods for the enhancement in PG production by $A$. sojae was demonstrated. This resulted in the generation of mutants with increased pectinolytic activities, which can be potentially employed in biotechnological processes for pectinase production either under submerged or solid-state conditions. Moreover, the characterization of specific pectinolytic enzyme sets obtained by selected mutants plays a significant role in the degradation of complex plant polysaccharides, which has potential industrial importance for tailormade applications, e.g. in food processes such as carrot juice production utilizing preparations predominantly containing PG activity.

Acknowledgments The authors gratefully acknowledge the support and facilities provided by Jacobs University Bremen gGmbH. The research leading to these results has received funding from the project PGSYS/ETB-2008-44 and the People Programme of the European Union's Seventh Framework Programme PGSYS EXCHANGE / FP7PEOPLE-2010-IRSES. Furthermore, the authors are indebted to Dr. S. Diercks-Horn from Jacobs University Bremen gGmbH for reviewing this article and for helpful suggestions.

\section{References}

Adsul MG, Bastawde KB, Varma AJ, Gokhale DV (2007) Strain improvement of Penicillium janthinellum NCIM 1171 for increased cellulase production. Bioresour Technol 98:1467-1473

Antier P, Minjares A, Roussos S, Raimbault M, Viniegra-González G (1993) Pectinase-hyperproducing mutants of Aspergillus niger C28B25 for solid-state fermentation of coffee pulp. Enzym Microb Technol 15:254-260

Barrios-González J (2012) Solid-state fermentation: physiology of solid medium, its molecular basis and applications. Process Biochem 47: $175-185$

Benoit I, Coutinho PM, Schols HA, Gerlach JP, Henrissat B, de Vries RP (2012) Degradation of different pectins by fungi: correlations and contrasts between the pectinolytic enzyme sets identified in 
genomes and the growth on pectins of different origin. BMC Genomics 13:321

Bos CJ (1987) Induction and isolation of mutants in fungi at low mutagen doses. Curr Genet 12:471-474

Bradford MM (1976) A rapid and sensitive method for the quantitation of microgram quantities of protein utilizing the principle of protein-dye binding. Anal Biochem 72:248-254

Buyukkileci AO, Tari C, Fernandez-Lahore M (2011) Enhanced production of exo-polygalacturonase from agro-based products by Aspergillus sojae. BioResources 6:3452-3468

Crook N, Alper HS (2012) Classical strain improvement. In: Patnaik R (ed) Engineering complex phenotypes in industrial strains, 1st edn. Wiley-AIChE, Hoboken, pp 1-34

Del Cañizo AN, Hours RA, Miranda MV, Cascone O (1994) Fractionation of fungal pectic enzymes by immobilized metal ion affinity chromatography. J Sci Food Agric 64:527-531

De Nicolás-Santiago S, Regalado-González C, García-Almendárez B, Fernández FJ, Téllez-Jurado A, Huerta-Ochoa S (2006) Physiological, morphological, and mannase production studieson Aspergillus niger uam-gs1 mutants. J Biotechnol 9:51-60

Demain AL, Adrio JL (2008) Strain improvement for production of pharmaceuticals and other microbial metabolites by fermentation. In: Petersen F, Amstutz R (eds) Progress in drug research, v 65. Brinkhäuser Verlag, Basel, pp 253-289

Demir H, Göğüș N, Tari C, Heerd D, Fernandez Lahore M (2012) Optimization of the process parameters for the utilization of orange peel to produce polygalacturonase by solid-state fermentation from an Aspergillus sojae mutant strain. Turk J Biol 36:394-404

Durrands PK, Cooper RM (1988) Selection and characterization of pectinase-deficient mutants of the vascular wilt pathogen Verticillium albo-atrum. Physiol Mol Plant P 32:343-362

Galiotou-Panayotou M, Kapantai M, Kalantzi O (1997) Growth conditions of Aspergillus sp. ATHUM-3482 for polygalacturonase production. Appl Microbiol Biotechnol 47:425-429

Gögus N, Tari C, Oncü S, Unluturk S, Tokatli F (2006) Relationship between morphology, rheology and polygalacturonase production by Aspergillus sojae ATCC 20235 in submerged cultures. Biochem Eng J 32:171-178

Hadj-Taieb N, Ayadi M, Trigui S, Bouabdallah F, Gargouri A (2002) Hyperproduction of pectinase activities by a fully constitutive mutant (CT1) of Penicillium occitanis. Enzym Microb Technol 30:662-666

Heerd D, Yegin S, Tari C, Fernandez-Lahore M (2012) Pectinase enzyme-complex production by Aspergillus spp in sold-state fermentation: a comparative study. Food Bioprod Process 90:102-110

Heerikhuisen M, Van den Hondel C, Punt P (2005) Aspergillus sojae. In: Gellissen $\mathrm{G}$ (ed) Production of recombinant proteins. Novel microbial and eukaryotic expression systems. Wiley, Weinheim, pp 191-214
Jacob N (2009) Pectinolytic enzymes. In: Nigam PS, Pandey A (eds) Biotechnology for agro-industrial residues utilization. Springer, Dordrecht, pp 383-396

Kashyap DR, Vohra PK, Chopra S, Tewari R (2001) Applications of pectinases in the commercial sector: a review. Bioresour Technol 77: 215-227

Lang C, Dörnenburg H (2000) Perspectives in the biological function and the technological application of polygalacturonases. Appl Microbiol Biotechnol 53:366-375

Meireles LA, Guedes AC, Malcata FX (2002) Increase of the yields of eicosapentaenoic and docosahexaenoic acids by the microalga Pavlova lutheri following random mutagenesis. Biotechnol Bioeng 81:50-55

Mill PJ, Tuttobello R (1961) The pectic enzymes of Aspergillus niger. Biochem J 79:57-64

Naidu GSN, Panda T (1998) Production of pectolytic enzymes - a review. Bioprocess Eng 19:355-361

Nelson N (1944) A photometric adaption of the somogyi method for the determination of glucose. J Biol Chem 153:375-380

Panda T, Naidu GSN, Sinha J (1999) Multipleresponse analysis of pectinolytic enzymes by Aspergillus niger: a statistical view. Process Biochem 35:187-195

Parekh S, Vinci VA, Strobel RJ (2000) Improvement of microbial strains and fermentation processes. Appl Microbiol Biotechnol $54: 287-301$

Patil SR, Dayanand A (2006) Optimization of process for the production of fungal pectinases from deseeded sunflower hesd in submerged and solid-state conditions. Bioresour Technol 97: $2340-2344$

Patnaik R (2008) Engineering complex phenotypes in industrial strains. Biotechnol Prog 24:38-47

Sarkar S, Saunders G (1999) The role of genetics in the effective utilisation of filamentous fungi. IUPAC

Smith DC, Wood TM (1991) Isolation of mutants of Aspergillus awamori with enhanced production of extracellular xylanase and $\beta$ xylosidase. World J Microbiol Biotechnol 7:343-354

Ushijima S, Hayashi K, Murakami H (1982) The current taxonomic status of Aspergillus sojae used in Shoyu fermentation. Agric Biol Chem 46:2365-2367

Ustok FI, Tari C, Gogus N (2007) Solid-state production of polygalacturonase by Aspergillus sojae ATCC 20235. J Biotechnol 127:322-334

van den Brink J, de Vries RP (2011) Fungal enzyme sets for plant polysaccharide degradation. Appl Microbiol Biotechnol 91:14771492

Whitaker JR (1984) Pectic substances, pectic enzymes and haze formation in fruit juices. Enzym Microb Technol 6:341-349 